\title{
DRIVERS WITH DEMENTIA: ENVIRONMENT, ERRORS AND PERFORMANCE OUTCOMES
}

\author{
Angela Berndt, ${ }^{1}$ Esther May, ${ }^{1}$ Michael Clark $^{2}$ \\ ${ }^{1}$ University of South Australia \\ School of Health Sciences \\ Adelaide, South Australia \\ E-mail: angela.berndt@unisa.edu.au, esther.may@unisa.edu.au \\ ${ }^{2}$ Centre for Rehabilitation and Ageing Studies \\ Flinders University \\ Adelaide, South Australia
}

\begin{abstract}
Summary: The current non-experimental observational study adds to the body of evidence and literature by describing, from a person - environment - occupation model, the on-road performance of 115 licensed drivers who had dementia. The purpose is to potentially prescribe the essential criteria of environmental and driving tasks for on-road assessment inclusion.
\end{abstract}

\section{INTRODUCTION}

\section{Dementia}

Dementia describes the symptoms of a group of more than 200 illnesses or diseases that cause a progressive decline in mental functioning and cognition (Harvey, Fox \& Rosser, 1999). The prevalence of dementia is increasing, with a recent report showing that "in Australia there were over 162,000 people with dementia in 2002, including 6,600 under 65-with younger onset dementia," and that the "prevalence of dementia is growing rapidly" and is predicted to reach "the 500,000 mark around 2040" (Access Economics, 2003). Dementia is positively associated with ageing, and population prevalence increases with age from $0.077 \%$ at age $45-49$ to $23.6 \%$ $50 \%$ for ages 90 years and over (Corcoron, 2001). Although less common in younger age cohorts, dementia is not exclusively a condition of older people. Dementia progresses in phases; described as mild, moderate and severe delineated by a number of measures, including the Folsteins Mini Mental State Exam (MMSE) and The Washington University Clinical Dementia Rating (CDR) Scale, the latter of which has demonstrated high reliability and validity, and "requires a considerable amount of data to be collected both from the patient and from an informant" (Perneczky, Wagenpfeil, Komossa, Grimmer, Diehl \& Kurz, 2006) in order to describe functional deficits caused by dementia.

\section{Table 1. The MMSE to CDR equivalents}

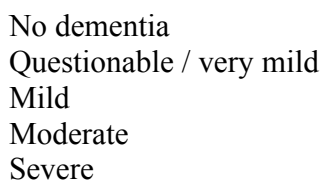

(Pernezcky et al. 2006)

$\begin{array}{ll}\text { MMSE } & \text { CDR } \\ 30 & 0 \\ 26-29 & 0.5 \\ 21-25 & 1 \\ 11-20 & 2 \\ 0-10 & 3\end{array}$




\section{Dementia and driving}

Dementia poses a risk to the performance of competent driving. Drivers with dementia are reported to crash more often than other cohorts once dementia advances past the very mild stages (Drachman \& Swearer, 1993), yet even in the early part of the syndrome impairment to attention is of concern (Duchek, Hunt, Ball, Buckles \& Morris, 1998). Impaired attention, judgement, memory, information processing speed, visuo-spatial perception and lack of insight into impairment reduce the driver's capacity to respond in a timely manner to important environmental cues (Lloyd, Cormack, Blais, Messeri, McCallum, Spicer, \& Morgan, 2001). High or increasing cognitive load may disable visual scanning capacity (Engstrom, Johansson \& Ostlund, 2005) and hence, complexity in the environment is a crucial factor in the relationship between declining cognitive capacity and driving competence.

Accident studies that describe the on-road outcomes of driving errors have contributed to an understanding of the environmental demands that the driver with dementia is likely to find confusing, and too-demanding in performance terms, by noting where crashes most often occur. Tasks that require constructional spatial awareness, such as positioning a car on the roadway or changing lanes, and complex visual information processing, such as making complex turns or stopping in a timely manner for traffic signals, are most difficult for the driver with dementia. The contributing factors to crashes involving complex right turns at traffic lights or multiple lane roadways are observation, attention and perception errors, as well as slowed motor performance (Keskinen, Ota \& Katila, 1998) and functional decline (McGwin \& Brown, 1999).

Consensus, reached by a group of expert researchers in 1994 states that every driver with dementia will need to cease driving at some point in the progression of the disease (Lundberg, Johansson, Ball, Bjerre, Blomqvist, Braekhus, et al., 1997). When dementia is assessed at a CDR of 2 or 3 , it is deemed to "be considered sufficient to warrant a recommendation of immediate cessation of driving" (Lundberg et al., 1997; Johansson \& Lundberg, 1997). This consensus has been extended to a "practice parameter" that individuals with advanced dementia should not drive (Dubinsky, Stein \& Lyons, 2000). It is argued that it is more difficult to make such decisions about individuals who have mild dementia due to variability of functional capacity, and that there is the potential to discriminate against a group of drivers with no more risk than that tolerated in other groups (Carr et al., 2000). Therefore, on-road and other forms of functional assessment of drivers with very mild to mild dementia are advocated (Carr, Duchek, \& Morris, 2000; Lundberg, 2003).

The potential outcome of declining driving capacity is the potential for an absent, incomplete or inappropriate action that leads to a driving error, and it is important to know if the driving errors of people with dementia are different from the unaffected driving population. Research conducted by Dobbs and colleagues in Canada is significant in its contribution to establishing the specific type of errors that drivers with dementia perform. Conducted with an aged matched control group, a younger control group and with drivers who have dementia, Dobbs et al. (1998) describe two classifications of errors: "criterion errors," which stand the groups apart and identify the driver with cognitive impairment, and "non-criterion" or the common less risky errors of experienced drivers. Within the two Dobbs et al. classifications are 13 error types-five in the criterion (hazardous, minor positioning, overcautiousness, turn positioning, and scanning 
errors) and seven in the non-criterion categories. A hazardous error category was developed for errors which included exclusively a clearly dangerous error regardless of the type of manoeuvre involved, and involved intervention from the driving instructor or accommodation from other road users. The error types indistinguishable between the groups were road-code and rules-based actions, which were potentially correctable and not deemed as indicators of declining competence, including stop-position errors, speed errors, signal errors and vehicle control (shaky steering or one-handed steering).

The Washington University Road Test validation study also showed that in comparison to healthy older drivers, drivers with dementia had lower average drive scores (on the test) and demonstrated more errors in the complex part of the test, demonstrated slower speed, had more difficulty judging the distance of oncoming traffic when turning, showed poor scanning, poor lane position control, were less aware of other vehicles and demonstrated more frequent and unexpected braking (Hunt et al., 1997). Di Stefano and Macdonald (2003) described the relationship among on-road errors, medical condition and on-road test outcome from a file audit study of 533 licensing authority road tests. In this Australian study, test outcome was "almost invariably determined by whether the driving authority testing officer needed to intervene to maintain safety." In the prospective on-road Dobbs study, 50\% of hazardous errors occurred while changing lanes, merging or negotiating an intersection, while the Di Stefano and Macdonald study (2003) reports a rate of 56\% in "identical circumstances."

\section{METHOD}

\section{Participants}

One hundred and seventeen drivers ( 87 male and 30 female) aged between 48 - 88 years, with clinically diagnosed cognitive impairment and who met visual guidelines for fitness to drive, had no co-morbidities, held a current unrestricted drivers license, and lived in a metropolitan area were recruited from a Memory Disorders Clinic as part of a wider neuro-psychology tests and driving outcome comparative study (see Clark, 2000). Two driver's results were excluded from data analysis as it was not possible to proceed to the open road route due to extreme safety concerns.

\section{Data collection}

The assessors were a driver trained occupational therapist (OTDA) and a professionally accredited driving instructor (DI). Both assessors were aware the driver had a cognitive impairment, but were blinded to the severity score and diagnostic category. Each person participated in a pre-assessment interview and commenced with a familiarisation drive conducted within a transport authority private roadway. The full assessment was conducted over an open road set route including consistent instruction and process, designed from occupational therapy grading principles and from research of relevant literature regarding at-risk drivers, with specific reference to the Washington University Road Test (Hunt, 1997).

The duration of the assessment was 60 minutes and it included 110 driving tasks. Performance on each task was nominally scored as $1=$ yes or $0=$ no when action-based (e.g., indicator 
applied correctly), or $1=$ safe and $0=$ unsafe when hazard perception / response-based (e.g., applied brake in response to hazard). Field notes recorded qualitative data and situational descriptions (e.g., comments made by the driver, the presence of unexpected events or environmental changes or the actions of other road users). The OTDA collected data and on conclusion of the drive, the OTDA \& DI discussed data and results of the assessment and formed a consensus on performance observations. The assessment resulted in a pass or fail outcome.

\section{Analysis}

The on-road data was analysed in binary by the pass or fail outcome for each driver by each task (115 drivers x 110 assessment tasks). Unpaired t tests at 95\% CI were calculated for age and MMSE for pass / fail groups. Grubbs analysis for outliers was performed identifying one female driver, aged 48, who was an outlier only for age. Field notes content analysis was conducted to identify error types and frequencies. Error definitions were determined and Fisher's Exact Tests and Odds Ratios 2 by 2 contingency tables were calculated for error type by location.

\section{RESULTS}

\section{Person characteristics}

Gender was not associated with passing or failing the on-road assessment. Both advancing age (mean pass group 73.6; mean fail group 77.2; p 0.0043) and the severity of dementia (mean MMSE pass group 25; mean MMSE fail group 22; $\mathrm{p}$ 0.0001) were significantly associated with the fail outcome.

\section{Error types}

There was statistically significant variability of performance within this group of drivers with dementia. The mean error rate for the pass group was $n=27$, while the mean error rate for the fail group was $n=54$ ( $p$ 0.0001). Thirteen error categories, based on Dobbs (1998), were analysed for statistically significant differences between the drivers who passed and failed the assessment. The errors that differentiated between the pass and fail group were mirror observation lapses, hazardous lapses, turn positioning, speed above set limit, purposefully risky / aggressive manoeuvres, rolled stop at stop signs, poor vehicle control (accurate gear selection, clutch and brake management) and incorrect stop positioning. A hazardous error was defined as any error that required the driving instructor to intervene either physically or verbally or that required other road users to take evasive action or slow unnecessarily. DI interventions included taking the steering wheel to guide the path of the vehicle, application of the dual safety brake, stating "caution here" or provision of verbal guidance to fuller attention and problem solving. The usual causes of DI intervention were crossing the midline into oncoming traffic flow, turning into the incorrect side of the road or attempting to travel in the incorrect direction, driving as the "crow flies" rather than following lane lines, failure to slow sufficiently for slowing devices such as speed humps or chicanes and failure to respond correctly to either green or red traffic signals and Stop signs, give-ways signs or railways crossings. Of the 110 task locations, 31 were statistically different in error frequency between the pass and fail groups. 
Those errors that did not differentiate between the groups were left / right scanning behaviours, signal lapses, lack of blind spot (shoulder) checks, minor positioning (poor parking) and overcautiousness (driving below limit or give way unnecessarily). That is, these errors were committed in similar numbers by both the fail and pass groups.

Task / environments

Table 2. Hazardous error and environmental locations

\begin{tabular}{|c|c|}
\hline Task type & $\begin{array}{l}\text { Odds ratio } \\
\text { (fail > pass) }\end{array}$ \\
\hline 1. U turn & 42 \\
\hline 2. Traffic signal $>$ choose lane $>$ dog leg turn & 36 \\
\hline 3. $\quad$ Right lane change & 28 \\
\hline 4. $\quad$ Speed $>$ mirror check $>$ positioning & 24 \\
\hline 5. $\quad$ Speed & 19 \\
\hline 6. $\quad$ Speed $>$ mirror check $>$ positioning & 19 \\
\hline 7. Lane change (left or right) & 19 \\
\hline 8. $\quad$ Brake without mirror check & 16 \\
\hline 9. $\quad$ Left turn $>$ right lane change & 15 \\
\hline 10. Traffic signal $>$ choose lane $>$ right turn with arrow & 15 \\
\hline 11. Traffic signal $>$ choose lane $>$ right turn with arrow & 15 \\
\hline 12. Straight at roundabout & 13 \\
\hline 13. Left turn with care & 13 \\
\hline 14. Left run with traffic (exit car park) & 13 \\
\hline 15. Left turn at give way sign & 11 \\
\hline 16. Lane position with mirror check & 11 \\
\hline 17. Right turn & 11 \\
\hline 18. Left turn with traffic signal arrow & 11 \\
\hline 19. Traffic signal $>$ choose lane $>$ left turn & 11 \\
\hline 20. Traffic signal $>$ choose lane $>$ left turn with arrow & 11 \\
\hline 21. Straight at roundabout & 10 \\
\hline 22. Right lane change & 10 \\
\hline 23. Right turn against traffic & 9 \\
\hline 24. Right turn against traffic & 9 \\
\hline 25. Left turn at give way sign & 9 \\
\hline 26. Stop sign & 9 \\
\hline 27. Red traffic signal & 9 \\
\hline 28. Lane position with mirror check & 9 \\
\hline 29. Red traffic signal & 6 \\
\hline 30. Speed humps & 5 \\
\hline 31. Right turn at $\mathrm{T}$ junction & 4 \\
\hline
\end{tabular}

There were statistically different results between the pass and fail groups for each of the thirteen error types and task locations. In this paper only hazardous errors will be highlighted. At the 31 / 110 statistically different assessment task locations (Fishers exact test), Odds Ratios analysis was conducted. At these locations, the fail-group drivers were between 42 to 4 times more likely to commit an error than the pass group. The results of the most significant hazardous error locations are presented in Table 2 . 


\section{CONCLUSION}

The results of this prospective on-road observational descriptive study support previous research. Consistently defined error types can be observed and recorded while conducting an on-road assessment with drivers with dementia. Within the dementia-affected population, as with nonimpaired drivers, common driver-habit-related error such as signal lapses and poor parking capacity is demonstrated. These "non-criterion" errors did not differentiate between pass or fail outcomes, however, "criterion" errors such as hazardous or speed errors (Dobbs et al., 1998) did differentiate between and the pass or fail outcomes. These results support previous literature that stresses assessment must be sensitive and decrease the potential for discriminatory assessment practices between healthy aged or other functionally impaired drivers and those with dementia (Dobbs et al., 1998; Lundberg, 2003). This study also supports previous research by demonstrating that within the dementia population error performance varies, with the younger and less cognitively impaired drivers performing better than their older and more severely impaired peers. Regardless of gender, errors increased in both frequency and severity with advancing age and cognitive decline.

The study describes in objective detail the task / locations that are of most concern to the driver with dementia. These environmental locations should be used as a minimum criteria set for onroad assessment design. The next phase of this research will determine the manner in which the environmental tasks can be applied in geographically diverse settings and on-road assessment design.

\section{REFERENCES}

Access Economics. (2003). The Dementia Epidemic: Economic Impact and Positive Solutions for Australia. Alzheimer's Australia, Canberra, Australia: Author.

Carr, D.B., Duchek, J., \& Morris, J.C. (2000). Characteristics of Motor Vehicle Crashes of drivers with Dementia of the Alzheimer's Type. JAGS, 48, 18-22.

Clark, M., Hecker, J., Cleland, E., Field, C., Berndt, A., Crotty, M., \& Snellgrove, C. (2000). Dementia and Driving. Commonwealth Department of Transport and Regional Services. Australian Transport Safety Bureau. T99/ 0574.

Corcoran, M.A. (2001). Dementia. In B.R Bonder \& M.B. Wagner (Eds.), Functional Performance in Older Adults ( $2^{\text {nd }}$ ed). Philadelphia: F.A. Davis Company, 287-304.

Dobbs, A.R., Heller, R.B., \& Schopflocher, D. (1998). A Comparative Approach to Identify Unsafe Older Drivers. Accident Analysis and Prevention, 30(3), 363-370.

Drachman, D.A., \& Swearer, J.M., for the collaborative study group. (1993). Driving and Alzheimer's disease: the risk of crashes. Neurology, 43, 2448-2456.

Di Stefano, M., \& Macdonald, W. (2003). Assessment of older drivers: Relationships among onroad errors, medical conditions and test outcome. Journal of Safety Research.

Duchek, J.M., Hunt. L., Ball, K., Buckles, V., \& Morris, J.C. (1998). Attention and Driving Performance in Alzheimer's Disease. Journal of Gerontology: Psychological Sciences, $53 B(2), 130-141$. 
Engstrum, J., Johansson, E., \& Ostlund, J. (2005). Effects of visual and cognitive load in real and simulated motorway driving. Transportation Research, Part F, 97-120.

Harvey, R., Fox, N.C., \& Rosser, M.N. (1999). Dementia Handbook. London: Martin Dunitz Ltd.

Hunt, L.A., Murphy, C.F., Carr D., Duchek, J.M., Buckles, V. \& Morris, J.C. (1997). Reliability of the Washington University Road Test. Archives Neurology. 54, 707-712.

Hunt, L.A., Murphy, C.F., Carr, D., Duchek, J.M., Buckles, V., \& Morris, J.C. (1997). Environmental Cueing May Effect Performance on a Road Test for Drivers with Dementia of the Alzheimer's Type. Alzheimer's Disease and Associated Disorders, 11(Supp. 1), 13-16.

Keskinen, E., Ota, H., \& Katila, A. (1998) Older drivers fail in intersections: Speed discrepancies between older and younger male drivers. Accident Analysis and Prevention, 30(3), 323-330.

Johansson, K., \& Lundberg, C. (1997). The 1994 International Consensus Conference on Dementia and Driving: a brief report. Swedish National Road Administration. Alzheimer's Disease and Associated Disorders, 11(Supp 1), 62-69.

Lloyd, S., Cormack, C.N., Blais, K., Messeri, G., McCallum, M., Spicer, K., \& Morgan, S. (2001). Driving and dementia: A review of the literature. Canadian Journal of Occupational Therapy, 68, $149-156$.

Lundberg, C., Johansson, K., Ball, K., Bjerre, B., Blomqvist, C., Braekhus, A., Brouwer, W., Bylsma, F., Carr, D., Englund, L., Friedland, R., Hakamies-Blomqvist, L., Klemetz, G., O’Neill, D., Odenheimer, G., Rizzo, M., Schelin, M., Seideman, M., Tallman, K., Viitanen, M., Waller, P., \& Winblad, B. (1997). Dementia and driving: An Attempt at Consensus. Alzheimer's Disease and Related Disorders, 11(1), 28-37.

Lundberg, C. (2003). Older drivers with Cognitive Impairments: Issues of Detection and Assessment. Neurotec Department, Division of Clinical Geriatrics, Karolinska Institutet, Huddinge University Hospital, Stockholm, Sweden.

Perneczky, R., Wagenpfeil, S., Komossa, K., Grimmer, T., Diehl, J., \& Kurz, A. (2006). Mapping Scores Onto Stages: Mini Mental State Exam and Clinical Dementia Rating American Journal of Geriatric Psychiatry, 14, 139-144.

World Health Organization. (1992). The ICD-10 Classification of Mental and Behavioural Disorders. Clinical Descriptors and Diagnostic Guidelines. Geneva: World Health Organization, Geneva. 